\title{
Power Split Strategy for Fuel Cell Hybrid Electric System
}

\author{
D. Di Domenico*, C. Speltino and G. Fiengo \\ Dipartimento di Ingegneria, Università del Sannio Piazza Roma 21, Benevento - Italy \\ e-mail: domenico.didomenico@unisannio.it - carmelo.speltino@unisannio.it - gifiengo@unisannio.it \\ * Corresponding author
}

Résumé - Stratégie de séparation des flux de puissance pour un système électrique hybride à pile à combustible - Ce papier illustre une stratégie de gestion de puissance pour un système hybride composé d'une pile à combustible, d'une batterie et d'un convertisseur DC/DC. Dans le but d'équilibrer les flux de puissance entre la pile à combustible et la batterie et d'éviter les dégâts causés par une dépression d'oxygène dans le cathode de la pile, un contrôleur découplé est proposé. Ce contrôleur se compose de deux parties. La première, un régulateur proportionnel-intégral, commande le compresseur et, par conséquent, le flux d'oxygène fourni au cathode. La deuxième, un régulateur linéaire-quadratique, gère le courant demandé par la pile à combustible et la batterie. Pour estimer l'état de charge de la batterie, un filtre de Kalman étendu a aussi été conçu. Les performances de la stratégie ont été analysées en simulation avec un modèle de batterie du $310^{\mathrm{e}}$ ordre.

Abstract - Power Split Strategy for Fuel Cell Hybrid Electric System - The power management of a hybrid system composed of a fuel cell, a battery and a DC/DC power converter is developed. A decoupled control strategy is proposed, aimed at balancing the power flow between the stack and the battery and avoiding electrochemical damage due to low oxygen concentration in the fuel cell cathode. The controller is composed of two components. The first controller regulates the compressor, and as a consequence the oxygen supplied to the cathode, via a classic Proportional-Integral controller. The second controller optimally manages the current demanded by the fuel cell and battery via a linearquadratic control strategy acting on the converter. An Extended Kalman Filter is also designed in order to estimate the battery State of Charge. The closed-loop performance was tested in simulation using a 310th-order system model. 


\section{INTRODUCTION}

Low-temperature fuel cells are promising alternative energy conversion systems, thanks to their very high efficiency in converting the chemical energy of hydrogen into electrical energy $[6,14,19]$, and zero emissions [5, 12]. Unfortunately, hydrogen is not found directly in nature, and it needs to be produced. Several techniques are proposed for hydrogen generation $[6,16]$. As an example, using the reforming principles, petroleum by-products or methane may be used to get hydrogen, which could be distributed and used as an absolutely clean energy supply. Although the reforming process produces carbon dioxide, which is the principal cause of the greenhouse effect, its production can be strictly controlled and it could be not dispersed in the atmosphere. Another way to produce hydrogen is water electrolysis, utilizing alternative and renewable energy sources (i.e. nuclear, solar or aeolian energy). Apart from the challenges associated with hydrogen storage and distribution, the potential for clean and efficient use of hydrogen still warrants more research and development in the optimization of fuel cell automation.

One of the key features of the control system devoted to the management of the fuel cell is the supply of oxygen to the cathode $[18,19]$, which is a particularly difficult task during the high-frequency transient in power demand. When current is drawn from a fuel cell, the air supply system should quickly replace the reacted oxygen, otherwise oxygen starvation might damage the cathode electrode while limiting the power response from the stack. In a highpressure fuel cell, a compressor motor is used to provide the required air into the cathode through a manifold $[13,19]$.

To prevent starvation, corrective actions such as rate limiters and reference governors for the fuel cell current can be employed $[18,19]$.

In [18] a load governor for starvation prevention has been proposed. It controls the current drawn from the fuel cell to ensure that constraints on the oxygen level are fulfilled. Basically, the governor acts as a dynamic filter preceding the closed-loop system which delays the current command slightly but preserves the disturbance rejection of the linear system. Although the performance of the reference governor can be suboptimal as compared with other controllers, the computational implementation can be much simpler, which is a significant advantage. The main limitation of this technique is the management of the power demand supply [19].

To avoid starvation and simultaneously provide an arbitrary level of power request, i.e. current demand, it is convenient to add a rechargeable auxiliary power source which can respond quickly to the increase in current demand.

A battery or an ultracapacitor should be an appropriate extra-power source. Both battery and ultracapacitor also appear capable of guaranteeing good vehicle performance and good fuel economy. In this paper, we choose a Fuel Cell

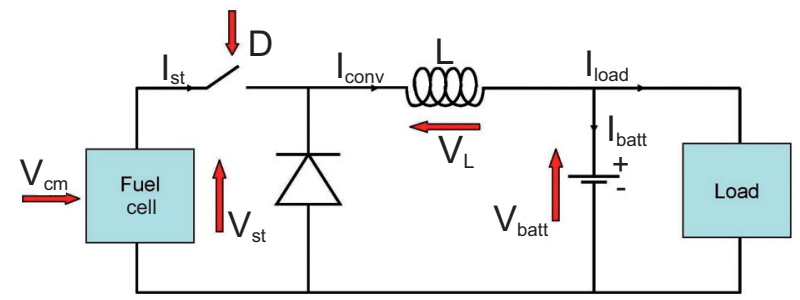

Figure 1

Fuel Cell Hybrid Power System. $v_{c m}$ is the compressor voltage; $v_{s t}$ and $I_{s t}$ are the voltage and current fuel cell; $D$ is the converter duty cycle; $I_{c o n v}$ is the converter current; $v_{L}$ is the inductance voltage; $v_{\text {batt }}$ and $I_{\text {batt }}$ are the voltage and current battery; $I_{\text {load }}$ is the current requested by the load.

Hybrid Power System (FCHPS) composed of a fuel cell and battery.

Furthermore, a DC/DC power converter is placed between them (see Fig. 1) to optimize the power flow between the fuel cell and the battery in order to satisfy the load power requirements, while ensuring the operation within any limitations of the electrochemical components such as battery over-charge/over-discharge and fuel cell current limit [8].

In the literature, several configurations of hybrid fuel cell systems are proposed [6-8, 10, 19]. In particular, in [19] a model predictive control of a fuel cell and a small capacitor hybrid system is proposed to avoid oxygen starvation. In [17] a load-following fuel cell system equipped with a compressor and a DC/DC converter is analyzed and modelbased techniques to tune two separate controllers for the compressor and the converter are shown. In $[8,10,11]$ a hybrid system fuel cell-battery is proposed. Different control techniques are illustrated depending on the specific aim, i.e. to minimize the hydrogen consumption [11] or to preserve the battery State of Charge $(S O C)$ and to limit the stack overpotential and voltage drops $[8,10]$.

In the following we propose a decoupled control strategy aimed at balancing the power flow between the stack and the battery, avoiding electrochemical damage of the fuel cell. In particular, we regulate the input to the motor that drives the fuel cell air flow compressor, and as a consequence the oxygen supplied to the cathode, via a classic Proportional Integral (PI) controller. We then optimally manage the current demanded to the fuel cell and battery via a Linear Quadratic (LQ) control strategy acting on the converter.

In the paper, we first describe the model used for the simulations. Then we introduce the simplified converterbattery model used for the controller design and we illustrate the adopted control strategy. Simulation results and some conclusions end the paper. 


\section{THE MODEL}

In the following, the models we used to test the proposed control architecture are described. We want to highlight that the fuel cell models are taken from the recent literature and our contribution is to model the battery and how these systems are connected, and mainly the adopted control strategy aimed at the power management of the whole system.

\subsection{Fuel Cell}

Thanks to its high power density, solid electrolyte, low corrosion and long stack life, the most promising and developed fuel cell technology for automotive applications is the Proton Exchange Membrane (PEM) fuel cell [12, 14, 16]. In the literature several PEM fuel cell models are proposed, each aimed at modeling a particular aspect based on the specific goal. As an example, a static model is proposed in [7] where the steady-state behavior of the fuel cell is described via a characteristic curve of cell voltage function of the current density. In our work, to correctly analyze the transient we used a complete fuel cell reactant model introduced by Pukrushpan, Peng and Stefanopoulou in [12]. The dynamic equations are modeled via a high non-linear function of the state, the compressor voltage $v_{c m}$ and the fuel cell current $I_{s t}$

$$
\dot{x}_{f c}=f_{f c}\left(x_{f c}, v_{c m}, I_{s t}\right)
$$

This is a 9th-order model, whose state variables are

$$
\begin{gathered}
x_{f c}=\left[\begin{array}{lllllll}
m_{\mathrm{O}_{2}} & m_{\mathrm{H}_{2}} & m_{\mathrm{N}_{2}} & \omega_{c p} & \ldots \\
\ldots & \ldots \\
\ldots & m_{s m} & m_{s m} & m_{w, a n} & m_{w, c a} & p_{r m}
\end{array}\right]^{T}
\end{gathered}
$$

where: $m_{\mathrm{O}_{2}}, m_{\mathrm{N}_{2}}$ and $m_{\mathrm{H}_{2}}$ are, respectively, the cathode oxygen and nitrogen mass and the anode hydrogen mass; $\omega_{c p}$ is the compressor speed; $p_{s m}$ and $m_{s m}$ are the pressure and the inlet air mass in the supply manifold; $m_{w, a n}$ and $m_{w, c a}$ are the anode and cathode water mass; $p_{r m}$ is the return manifold pressure.

For details on the non-linear equations and the constant parameter values see $[12,13]$. The model parameters were adjusted to assure a maximum power generation of $75 \mathrm{~kW}$, with a nominal stack voltage of $300 \mathrm{~V}$ and a nominal current of $250 \mathrm{~A}$.

Starting from compressor voltage and stack current, the model computes the fuel cell voltage, reproducing analytically the air and the hydrogen flows through the fuel cell system components. The compressor, supply manifold, cooler and humidifier are modeled for the air flow path. The hydrogen reaches the stack through its humidifier. The voltage is calculated as a function of stack current, cathode pressure, reactant partial pressures, temperature and membrane humidity. Its open circuit value is calculated from the energy balance between chemical reactant energy and electrical energy, considering the activation, ohmic and concentration losses.

The model outputs are the stack voltage $v_{s t}$ and the compressor air flow rate $W_{c p}$. At steady state, the compressor air flow needs to satisfy the desired oxygen excess ratio, $\lambda_{\mathrm{O}_{2}}$, based on the following relation:

$$
W_{c p}=\frac{n M_{\mathrm{O}_{2}}}{4 F} \frac{1+\omega_{a t m}}{x_{\mathrm{O}_{2}, a t m}} \lambda_{\mathrm{O}_{2}} I_{s t}
$$

where $n$ is the number of the stack elementary cells, $M_{\mathrm{O}_{2}}$ the oxygen molar mass, $F$ the Faraday's constant, $\omega_{\text {atm }}$ the humidity ratio and $x_{\mathrm{O}_{2} \text {,atm }}$ is the oxygen molar fraction in the atmospheric air drown in the fuel cell.

\subsection{Battery}

Many battery models with different complexity exist in the literature. Often a simple model with a specific electrical resistor and capacitor to reproduce the electrical properties of the battery connection is used $[5,8]$. Sometimes more complex models, i.e. obtained by modeling the kinetic of reactions and the diffusion phenomena [2] are presented.

In our work, a complete electrochemical model for a lithium-ion battery is adopted. Furthermore, an Extended Kalman Filter is used to estimate the battery State of Charge.

In order to mathematically model the battery, both macroscopic and microscopic behavior have to be considered. One can start with very basic microscopic equations, i.e. the conservation of mass and the conservation of charge equations for every species, and after that, try to obtain the macroscopic equations. The microscopic equations describe the battery system with 4 quantities, which are solid and electrolyte concentrations, and solid and electrolyte potentials. In addition, the microscopic current density will be defined via a function of all of them. These quantities depend on the time and the position along the cell. It is generally accepted that a microscopic description of these quantities should be impossible, due to the complexity of the interfaces [15]. So, macroscopic governing equations using the local volumeaveraging technique need to be derived.

The resulting equations describe the battery system with four quantities, i.e. solid and electrolyte concentrations $\left(c_{s}\right.$, $\left.c_{e}\right)$, and solid and electrolyte potentials $\left(\phi_{s}, \phi_{e}\right)$. The complete set of equations describing the micro-macroscopic model is $[15,20]$

$$
\begin{gathered}
i_{e}(x)=-\kappa^{e f f} \vec{\nabla}_{x} \phi_{e}-\kappa_{D}^{e f f} \vec{\nabla}_{x} \ln c_{e} \\
i_{s}(x)=-\sigma^{e f f} \vec{\nabla}_{x} \phi_{s} \\
\vec{\nabla}_{x} i_{e}(x)=j^{L i} \\
\vec{\nabla}_{x} i_{s}(x)=-j^{L i} \\
\frac{\partial \epsilon_{e} c_{e}}{\partial t}=\vec{\nabla}_{x}\left(D_{e}^{e f f} \vec{\nabla}_{x} c_{e}\right)+\frac{1-t^{0}}{F} j^{L i}
\end{gathered}
$$


TABLE 1

Battery parameters

\begin{tabular}{|c|c|c|c|}
\hline Parameter & Negative electrode & Separator & Positive electrode \\
\hline Thickness $(\mathrm{cm})$ & $\delta_{n}=50 \times 10^{-4}$ & $\delta_{\text {sep }}=25.4 \times 10^{-4}$ & $\delta_{p}=36.4 \times 10^{-4}$ \\
\hline Particle radius $R_{S}(\mathrm{~cm})$ & $1 \times 10^{-4}$ & - & $1 \times 10^{-4}$ \\
\hline Active material volume fraction $\varepsilon_{s}$ & 0.580 & - & 0.500 \\
\hline Electrolyte phase volume fraction (porosity) $\varepsilon_{e}$ & 0.332 & 0.5 & 0.330 \\
\hline $\begin{array}{l}\text { Conductivity of solid active material } \\
\sigma\left(\Omega^{-1} \mathrm{~cm}^{-1}\right)\end{array}$ & 1 & - & 0.1 \\
\hline Effective conductivity of solid active material & $\sigma^{e f f}=\varepsilon_{s} \sigma$ & - & $\sigma^{e f f}=\varepsilon_{s} \sigma$ \\
\hline Transference number $t_{+}^{0}$ & 0.363 & 0.363 & 0.363 \\
\hline $\begin{array}{l}\text { Electrolyte phase ionic conductivity } \\
\kappa\left(\Omega^{-1} \mathrm{~cm}^{-1}\right)\end{array}$ & $\kappa=0.0158 c_{e} \exp \left(0.85 c_{e}^{1.4}\right)$ & $\kappa=0.0158 c_{e} \exp \left(0.85 c_{e}^{1.4}\right)$ & $\kappa=0.0158 c_{e} \exp \left(0.85 c_{e}^{1.4}\right)$ \\
\hline Effective electrolyte phase ionic conductivity & $\kappa^{e f f}=\left(\varepsilon_{e}\right)^{1.5} \kappa$ & $\kappa^{e f f}=\left(\varepsilon_{e}\right)^{1.5} \kappa$ & $\kappa^{e f f}=\left(\varepsilon_{e}\right)^{1.5} \kappa$ \\
\hline Effective electrolyte phase diffusional conductivity & $\kappa_{D}^{e f f}=\frac{2 R T \kappa^{e f f}}{F}\left(t_{+}^{0}-1\right)$ & $\kappa_{D}^{e f f}=\frac{2 R T \kappa^{e f f}}{F}\left(t_{+}^{0}-1\right)$ & $\kappa_{D}^{e f f}=\frac{2 R T \kappa^{e f f}}{F}\left(t_{+}^{0}-1\right)$ \\
\hline $\begin{array}{l}\text { Electrolyte phase diffusion coefficient } \\
D_{e}\left(\mathrm{~cm}^{2} \mathrm{~s}^{-1}\right)\end{array}$ & $2.6 \times 10^{-6}$ & $2.6 \times 10^{-6}$ & $2.6 \times 10^{-6}$ \\
\hline Effective electrolyte phase diffusion coefficient & $D_{e}^{e f f}=\left(\varepsilon_{e}\right)^{1.5} D_{e}$ & $D_{e}^{e f f}=\left(\varepsilon_{e}\right)^{1.5} D_{e}$ & $D_{e}^{e f f}=\left(\varepsilon_{e}\right)^{1.5} D_{e}$ \\
\hline Solid phase diffusion coefficient $D_{s}\left(\mathrm{~cm}^{2} \mathrm{~s}^{-1}\right)$ & $2.0 \times 10^{-12}$ & - & $3.7 \times 10^{-12}$ \\
\hline $\begin{array}{l}\text { Maximum solid phase concentration } \\
c_{S, \max }\left(\mathrm{mol} \mathrm{cm}^{-3}\right)\end{array}$ & $16.1 \times 10^{-3}$ & - & $23.9 \times 10^{-3}$ \\
\hline Average electrolyte concentration $\bar{c}_{e}\left(\mathrm{~mol} \mathrm{~cm}^{-3}\right)$ & $1.2 \times 10^{-3}$ & $1.2 \times 10^{-3}$ & $1.2 \times 10^{-3}$ \\
\hline Change transfer coefficients $\alpha_{a}, \alpha_{c}$ & $0.5,0.5$ & - & $0.5,0.5$ \\
\hline $\begin{array}{l}\text { Active surface area per electrode unit volume } \\
a_{S}\left(\mathrm{~cm}^{-1}\right)\end{array}$ & $a_{s_{n}}=\frac{3 \varepsilon_{e}}{R_{S}}$ & - & $a_{s_{p}}=\frac{3 \varepsilon_{e}}{R_{S}}$ \\
\hline Electrode plate area, $A\left(\mathrm{~cm}^{2}\right)$ & 10452 & - & 10452 \\
\hline
\end{tabular}

$$
\frac{\partial c_{s}}{\partial t}=\vec{\nabla}_{r}\left(D_{s} \vec{\nabla} c_{s}\right)
$$

with the Butler-Volmer current density

$$
j^{L i}(x)=a_{s} j_{0}\left[\exp \left(\frac{\alpha_{a} F}{R T} \eta\right)-\exp \left(-\frac{\alpha_{c} F}{R T} \eta\right)\right]
$$

where the overpotential $\eta$ is obtained as

$$
\eta=\phi_{s}-\phi_{e}-U\left(c_{s e}\right)
$$

and $U$ is the open circuit voltage, a function of the solid concentration at the electrolyte interface indicated with $c_{s e}(x, t)=c_{s}\left(x, R_{s}, t\right)$. The variables $R$ and $F$ are the universal gas and the Faraday's constants and $T$ is the absolute temperature. The coefficient $j_{0}$ in Equation (9) also exhibits a modest dependence on the solid and electrolyte concentration, according to

$$
j_{0}=\left(c_{e}\right)^{\alpha_{a}}\left(c_{s, \max }-c_{s e}\right)^{\alpha_{a}}\left(c_{s e}\right)^{\alpha_{c}}
$$

The model parameters are summarized in Table 1 . In [3] and [4] the partial differential Equations (3-8) are discretized into 300th-order Ordinary Differential Equations. A reduced-order model is also introduced in [3] and used to design an Extended Kalman Filter for the battery State of Charge estimation. For the sake of brevity, these details will not be reported here.

For the purposes of this paper, the battery parameters were adjusted in order to ensure a nominal voltage of $120 \mathrm{~V}$ and a maximum current of $50 \mathrm{~A}$, fixing the hybridization degree (HD), i.e. the ratio between the nominal power generated by the two sources

$$
H D=\frac{P_{b a t t}}{P_{b a t t}+P_{f c}}
$$

to be equal to 0.07 . The battery nominal capacity was chosen equal to $10 \mathrm{Ah}$.

\subsection{DC/DC Converter}

The fuel cell and the battery model are coupled via a DC/DC converter which manages the current from the stack and the battery. Typically, a DC/DC converter is a device that accepts a DC input voltage and produces a lower or higher DC output voltage.

Here, in the proposed FCHPS, the standard electric configuration of the converter is modified after substituting the capacitor of the DC/DC converter with the battery. The inputs are now the stack and the battery voltages and the outputs are the stack and the battery currents. The controller acts on the interrupt (see Fig. 1), regulating the average value of the fraction of time that the converter is conducting, i.e. the interrupt is switched on. This average value is generally indicated as the duty cycle $D$ [17], and it is considered as the control input to the system. When the controller drives the interrupt in the state $\mathrm{ON}$, the fuel cell is connected to the 


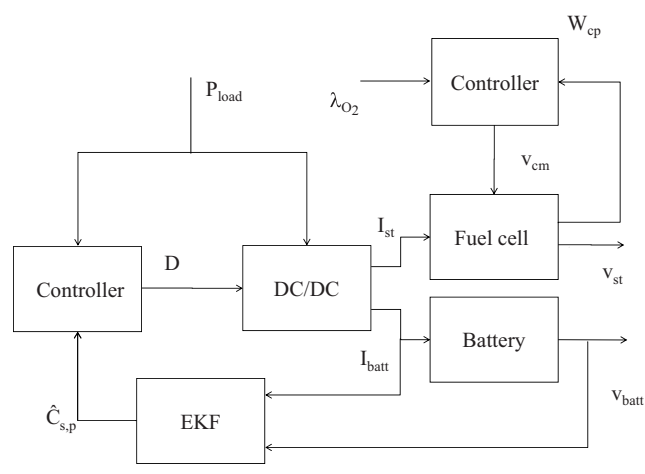

Figure 2

Decoupled control scheme. Some connections are omitted for the sake of readability.

load and provides power both to the battery and the load. Conversely, if the interrupt is OFF, the demanded power is provided exclusively by the battery. So, acting on the duty cycle it is possible to determine the average distribution of the power load between the two energy sources power balancing the load current on the battery and stack. Now, considering as other inputs to the DC/DC the requested load power $P_{\text {load }}$, the dynamic model can be obtained according to

$$
\dot{I}_{\text {conv }}=\frac{1}{L}\left(D v_{s t}-v_{\text {batt }}\right)
$$

where the state $I_{\text {conv }}$ is the converter current. Finally, the converter outputs are the currents towards the fuel cell and the battery, as follows

$$
\begin{gathered}
I_{s t}=D I_{\text {conv }} \\
I_{\text {batt }}=I_{\text {conv }}-\frac{P_{\text {load }}}{v_{\text {batt }}}
\end{gathered}
$$

\section{THE CONTROL STRATEGY}

The control objective is to provide the requested power to the load while regulating the battery State of Charge and the oxygen ratio of the fuel cell cathode at their nominal values, acting on the converter duty cycle $D$ and the compressor voltage $v_{c m}$. The problem can be solved with a decoupled control architecture, shown in Figure 2. The air flow controller is designed to regulate the oxygen ratio $\lambda_{\mathrm{O}_{2}}$, feeding back the compressor air flow rate and acting on the compressor voltage. This controller can reach high performance if the stack current is constant or changes slowly. Then, a second controller, working on the converter, optimally regulates the battery State of Charge, guaranteeing at the same time the requested power (or current to the load) and avoiding the

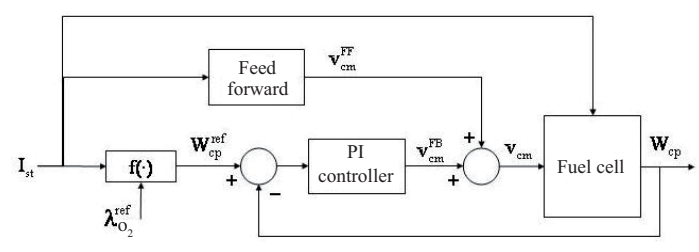

Figure 3

Fuel cell control scheme.

fast transient of the stack current. In particular, it commands the battery to compensate for the power request during the fast transients while using the fuel cell to furnish the desired power and recover the State of Charge.

In the following subsections the controllers are described in detail.

\subsection{Fuel Cell Operating Point Regulation}

The key feature in fuel cell control is the regulation of the oxygen ratio at the cathode in order to prevent oxygen starvation and, as a consequence, the performance and the potential life reduction $[18,19]$. The main problem is that $\lambda_{\mathrm{O}_{2}}$ is not measurable, but we can estimate it by measuring $W_{c p}$ and inverting Equation (2), that approximates the relationship between $\lambda_{\mathrm{O}_{2}}$ and $W_{c p}$ at steady state. Unfortunately, during the current transients, the error introduced by this approximation propagates to the regulation of the oxygen ratio, causing an inevitable performance reduction. Hence, it is critical that the stack current is constant or varies slowly, which will be guaranteed with the DC/DC converter controller.

Here, the adopted control strategy is obtained by combining a feedback and feedforward action, as shown in Figure 3. The first is realized via a PI controller, whose input is the error between the measurement of $W_{c p}$ and reference $W_{c p}^{r e f}$. This function of the actual stack current is computed by (2) where the oxygen ratio is substituted with its reference value $\lambda_{\mathrm{O}_{2}}^{\text {ref }}=2$.

The feedforward controller is a static function relating the compressor voltage to the stack current at optimal oxygen ratio. This function was calculated by linearization, approximating the state equations with first-order Taylor series. The result, according to

$$
v_{c m}^{F F}=20.16+0.712 I_{s t}
$$

was confirmed by using the full non-linear simulation.

\subsection{Power Management}

The main component of the proposed architecture is the controller, devoted to balancing the requested power between 


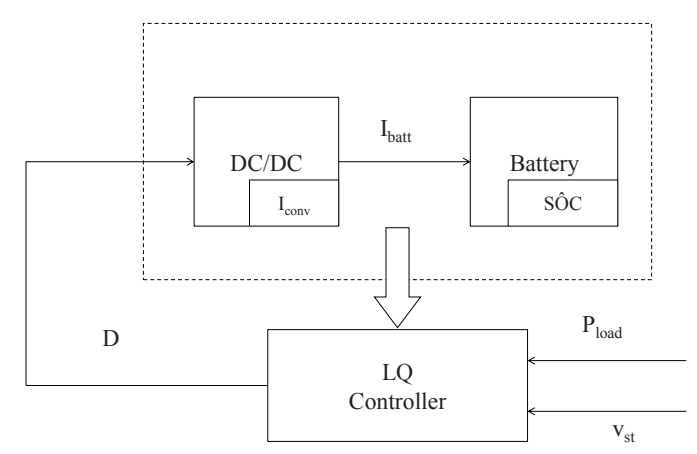

Figure 4

Cell open circuit voltage function of SOC. The curve was obtained using the full-order battery model.

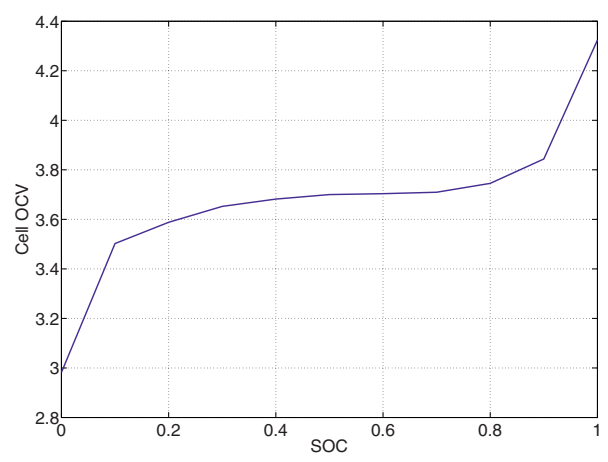

Figure 5

Power management control. Schematic diagram of the lees. The inputs of the LQR are the state of the simplified system, i.e. the measurement $I_{\text {conv }}$ and the EKF estimation of the SOC, and the parameters $P_{\text {load }}$ and $v_{s t}$.

the fuel cell and the battery. The objectives are to regulate the optimal State of Charge and minimize the rate of variation in the stack current, subject to the constraint on power demand $P_{\text {load }}=v_{\text {batt }} I_{\text {load }}$. The main idea is to furnish the total load current through the fuel cell at steady state, and to compensate with the battery during the fast transients.

To this aim, we propose an LQ-based control strategy designed on the DC/DC converter and a battery model (see Fig. 4).

In order to reduce the computational effort the controller is based on a very simplified battery model which describes the variation in the $S O C$ of the battery as a function of the demanded current $I_{\text {batt }}$. In particular, the charge stored or released by the battery is computed simply by integrating the battery current. Hence, the input of the model is the current, and the state is the $S O C$, whose derivative is computed by dividing the incoming current by the battery capacity $Q_{\max }$, as follows

$$
S \dot{O} C=\frac{I_{\text {batt }}}{Q_{\max }}
$$

The output is the voltage $v_{\text {batt }}$, determined by a non-linear static curve function of the SOC obtained using the complete cell model. The resulting curve is shown in Figure 5. It is to highlight that this simplified battery model does not predict the physical battery limitations, contrarily to the full-order electrochemical model used for the system simulation and for the Kalman Filter design. In particular, its performance decreases in correspondence to the highfrequency transients. Nevertheless, as will be pointed out in the simulation results, it appears to be enough to regulate the battery State of Charge and to correctly manage the power split while ensuring a feasible real-time system management. A second-order ODE is obtained by coupling the battery model with the DC/DC converter model as

$$
\begin{gathered}
\dot{I}_{\text {conv }}=-\frac{1}{L} v_{\text {batt }}(S O C)+\frac{1}{L} v_{s t}(t) D \\
S \dot{O} C=\frac{I_{\text {conv }}}{Q_{\max }}-\frac{1}{Q_{\max }} \frac{P_{\text {load }}(t)}{v_{\text {batt }}(S O C)}
\end{gathered}
$$

where $I_{\text {conv }}$ and $S O C$ form the state vector $x, D$ is the control input $u$, and $v_{s t}(t)$ and $P_{\text {load }}(t)$ are considered as time-varying parameters.

The objective function was selected after taking into account the goals both on the battery State of Charge and on the rate of variation in the stack current, as follows

$$
V=\frac{1}{2} \int_{0}^{\infty}\left[(x-\bar{x})^{T} Q(x-\bar{x})+\rho(u-\bar{u})^{2}\right] d t
$$

where $(\bar{x}, \bar{u})$ is the equilibrium point of system (18) related to the desired $S O C$ value, i.e. $\bar{x}_{2}=\overline{S O C}=80 \%$ and

$$
\begin{gathered}
\bar{x}_{1}=\bar{I}_{\text {conv }}=\frac{P_{\text {load }}}{\bar{v}_{\text {batt }}} \\
\bar{u}=\bar{D}=\frac{\bar{v}_{\text {batt }}}{v_{s t}}
\end{gathered}
$$

where $\bar{x}_{1}$ and $\bar{v}_{\text {batt }}=103.9 \mathrm{~V}$ the converter current and the battery voltage corresponding to $\overline{S O C}$.

We have to highlight that the inputs of the LQR are the state of the simplified system, i.e. the $I_{c o n v}$ and the SOC, and the parameters $P_{\text {load }}$ and $v_{s t}$. In particular, the battery State of Charge is obtained via the Extended Kalman Filter estimation, as described in the previous section.

In order to apply the well-known optimal LQ controller, the system (18) is linearized around the equilibrium point (20)

$$
\delta \dot{x}=A(t) \delta x+B(t) \delta u
$$

where $\delta x=x-\bar{x}$ and $\delta u=u-\bar{u}$ are, respectively, the deviation of the state and the control input from the equilibrium point, and the time-varying matrices $A(t)$ and $B(t)$ are computed according to

$$
\begin{aligned}
& A(t)=\left(\begin{array}{cc}
0 & -\frac{1}{L} \frac{\partial \bar{v}_{\text {batt }}}{\partial S O C} \\
\frac{1}{Q_{\max }} & \frac{P_{\text {load }}(t)}{Q_{\text {max }} \bar{v}_{\text {batt }}^{2}} \frac{\partial \bar{v}_{\text {batt }}}{\partial S O C}
\end{array}\right) \\
& B(t)=\left(\begin{array}{c}
\frac{v_{s t}(t)}{L} \\
0
\end{array}\right)
\end{aligned}
$$


with $\frac{\partial \bar{v}_{\text {batt }}}{\partial S O C}$ the change of battery voltage with respect to the $S O C$ evaluated at the nominal $\overline{S O C}$.

Hence, the objective function (19) is related to the linearized system as follows

$$
V=\frac{1}{2} \int_{0}^{\infty}\left[\delta x^{T} Q \delta x+R \delta u^{2}\right] d t
$$

The values of the matrices $Q$ and $R$ were determined using the Matlab/Simulink "Genetic Algorithm and Direct Search Toolbox"' [9]. The quality index was the quadratic error on $\lambda_{\mathrm{O}_{2}}$ during a step from $30 \mathrm{~kW}$ to $35 \mathrm{~kW}$ in requested power, and the population size was set to 20 . The power split goal allows the management strategy to relax the regulation of the $S O C$ during the most fuel-cell-stressful manoeuvres, which translates to a larger value for $R$ than $Q$. The optimal values we found are $Q=\operatorname{diag}(10,0.143)$ and $R=10^{6}$. These values are also capable of assuring that the limitation on the range of variability in the $S O C$ and the duty cycle does not affect the control strategy closed-loop stability. The control law is

$$
\delta u^{*}=-R^{-1} B(t)^{T} P \delta x
$$

where $P$ is the solution of the Riccati equation. As usual, to avoid an excessive computational cost and to permit an online implementation, the suboptimal solution was adopted, obtained by solving the algebraic Riccati equation [1]

$$
P(t) A(t)+A(t)^{T} P(t)-P(t) B(t) R^{-1} B(t)^{T} P(t)+Q=0
$$

Finally, the control input can be obtained

$$
u^{*}=\bar{u}-R^{-1} B(t)^{T} P(x-\bar{x})
$$

\section{SIMULATION RESULTS}

The performance of the proposed control strategy was investigated through Matlab/Simulink. In order to reduce the computational effort during the Matlab/Simulink simulation, the Riccati matrix $P$ was considered constant until the coefficients of the (25), i.e. the matrices $A$ and $B$, change significantly. These matrices are a function of $P_{\text {load }}(t)$ and $v_{s t}(t)$, so two dynamic thresholds were set and the values of the Riccati matrix $P$ were updated when the variation in power request or stack voltage was greater then $1 \%$.

A large number of simulations was performed to assess the closed-loop performance and to test the strategy stability. Although the robustness property was not proved analytically, the Kalman Filter robustness was checked using numerical simulations. Stability and sensitivity were analyzed and the analysis results are summarized in Table 2. In particular, it was monitored that the strategy closed-loop stability and performance were not jeopardized by the filter modeling error. The percent error on SOC mean square error

\begin{tabular}{|c|c|c|}
\hline Parameter & Variation & $\%$ on SOC mean square error \\
\hline$c_{s_{\max , p}}$ & $\begin{array}{l}-20 \% \\
-10 \% \\
+10 \% \\
+20 \%\end{array}$ & $\begin{array}{l}\bar{e}_{\%}=1.1 \times 10^{-1} \\
\bar{e}_{\%}=4.32 \times 10^{-2} \\
\bar{e}_{\%}=3.86 \times 10^{-2} \\
\bar{e}_{\%}=7.36 \times 10^{-1}\end{array}$ \\
\hline$c_{s_{\max , n}}$ & $\begin{array}{l}-20 \% \\
-10 \% \\
+10 \% \\
+20 \% \\
\end{array}$ & $\begin{array}{l}\bar{e}_{\%}=1.75 \times 10^{-1} \\
\bar{e}_{\%}=7.87 \times 10^{-2} \\
\bar{e}_{\%}=6.44 \times 10^{-2} \\
\bar{e}_{\%}=1.16 \times 10^{-1}\end{array}$ \\
\hline$D_{s_{n}}$ & $\begin{array}{l}-20 \% \\
-10 \% \\
+10 \% \\
+20 \% \\
\end{array}$ & $\begin{array}{l}\bar{e}_{\%}=6.11 \times 10^{-3} \\
\bar{e}_{\%}=2.77 \times 10^{-3} \\
\bar{e}_{\%}=2.51 \times 10^{-3} \\
\bar{e}_{\%}=5.65 \times 10^{-3}\end{array}$ \\
\hline$a_{s p}$ & $\begin{array}{l}-20 \% \\
-10 \% \\
+10 \% \\
+20 \% \\
\end{array}$ & $\begin{array}{l}\bar{e}_{\%}=4.85 \times 10^{-2} \\
\bar{e}_{\%}=2.15 \times 10^{-2} \\
\bar{e}_{\%}=1.17 \times 10^{-2} \\
\bar{e}_{\%}=3.18 \times 10^{-2}\end{array}$ \\
\hline$a_{s_{n}}$ & $\begin{array}{l}-20 \% \\
-10 \% \\
+10 \% \\
+20 \%\end{array}$ & $\begin{array}{l}\bar{e}_{\%}=1.71 \times 10^{-1} \\
\bar{e}_{\%}=8.12 \times 10^{-2} \\
\bar{e}_{\%}=6.83 \times 10^{-2} \\
\bar{e}_{\%}=1.01 \times 10^{-1}\end{array}$ \\
\hline$A$ & $\begin{array}{l}-20 \% \\
-10 \% \\
+10 \% \\
+20 \% \\
\end{array}$ & $\begin{array}{l}\bar{e}_{\%}=2.89 \times 10^{-2} \\
\bar{e}_{\%}=1.22 \times 10^{-2} \\
\bar{e}_{\%}=1.02 \times 10^{-2} \\
\bar{e}_{\%}=1.99 \times 10^{-2}\end{array}$ \\
\hline $\begin{array}{l}c_{s_{\max , p}} \\
c_{s_{\max , n}} \\
\end{array}$ & & $\bar{e} \%=8.89 \times 10^{-1}$ \\
\hline
\end{tabular}
was computed by comparing the SOC estimation obtained
TABLE 2

Robustness analysis results

by the nominal filter and the SOC estimation obtained by a filter with the parameter error. Denoting with $S \hat{O} C_{n o m}(t)$ the nominal estimation and with $S \hat{O} C_{\text {par }}(t)$ the estimation with the parameter indetermination, the mean square error is computed as

$$
S O C_{M S E}=\int\left(S O \hat{O} C_{n o m}(t)-S \hat{O} C_{p a r}(t)\right)^{2} d t
$$

It has to be noted that each parameter was considered separately, apart from the last row of the table, that shows the percent error obtained by combining the two worst cases, i.e. the error on $c_{S_{\max , p}}$ and $c_{S_{\max , n}}$. Finally, Figure 6 shows the system controlled outputs to a step in current demand from $20 \mathrm{~kW}$ to $35 \mathrm{~kW}$ when $10 \%$ of uncertainness was introduced simultaneously on the more sensitive filter and controller parameters, i.e. $c_{s_{\max , p}}, c_{s_{\max , n}}, D_{s_{n}}, D_{s_{p}}, a_{s_{n}}$ and $a_{s_{p}}$.

Selected simulations in the Matlab/Simulink environment are used to demonstrate the strategy effectiveness. Figures 7 and 8 show the selected load profiles, from simulations 1 and 2 , which are comprised of steps in power demand. In particular, for simulation 1, during the first $250 \mathrm{~s}$ the power demand exhibits 5 steps, starting from a minimum value of $20 \mathrm{~kW}$ until a maximum value of $40 \mathrm{~kW}$. During the last $250 \mathrm{~s}$ a constant value $(25 \mathrm{~kW})$ was set for the power request in order to evaluate the performance of the strategy 

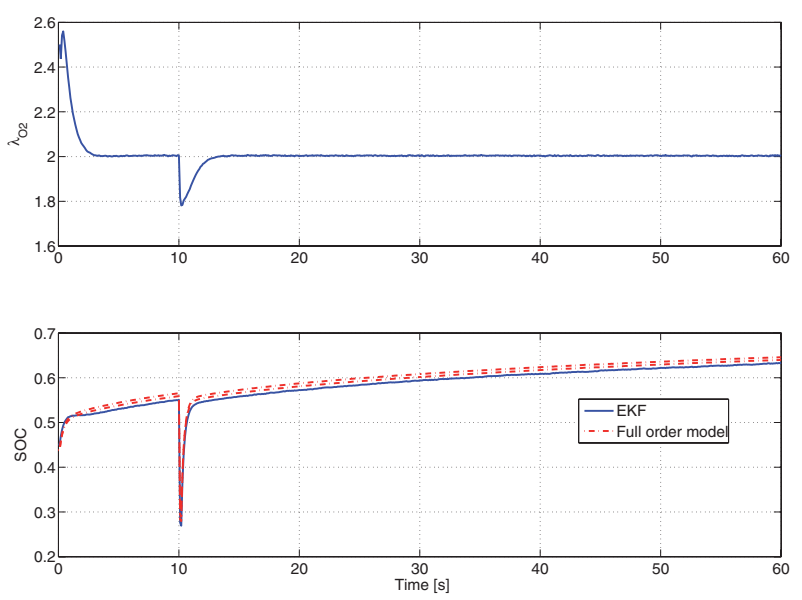

Figure 6

Robustness analysis: system controlled outputs to a step in power demand. An uncertainness of $10 \%$ affects the filter and the LQR parameters. The SOC is compared with the full-order model prediction.

in recharging the battery from $50 \%$ of the total capacity to the desired $S O C$ value $(S O C=80 \%)$. Simulation 2 tests the strategy under different operational conditions and takes into account the measurement uncertainties. Figures 9-11 summarize the simulation 1 results, while Figures 12-14 show the simulation 2 results. The first plot of Figure 9 shows that the oxygen ratio accurately reaches the desired value at steady state, and that the error quickly recovers during the fast power transient. The error on $\lambda_{\mathrm{O}_{2}}$ is mainly due to the approximation error associated with (2) during stack current transients, that propagates to the oxygen ratio regulation. This argument is confirmed by the second plot of Figure 11, that shows the performance of the controller aimed at the regulation of the compressor air flow rate. This control difficulty could be mitigated by a $\lambda_{\mathrm{O}_{2}}$ observer. Moreover, we want to highlight that a correct power split can support the action of the fuel cell controller in regulating the oxygen ratio and avoid an excessive complexity.

Figure 10 shows the performance of the power split strategy, namely the regulation of the battery State of Charge and the balancing of the power demand between the two sources. The response of Figure 10 also allows the evaluation of the steady-state closed-loop performance, showing that the fuel cell furnishes both the desired power and, mainly at the beginning, the extra power necessary to charge the battery. Indeed, the second plot of Figure 9 shows that the $S O C$ is approaching the desired value. Conversely, during the fast transients, the controller compensates with the battery the amount of power that the fuel cell cannot provide, in order not to degrade its performance. In particular, the zoom compares the $S O C$ estimated by the filter with the maximum and

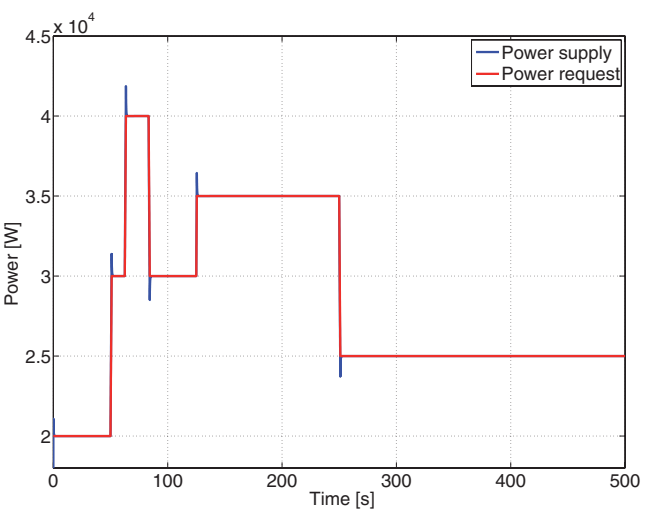

Figure 7

Simulation 1: requested load power.

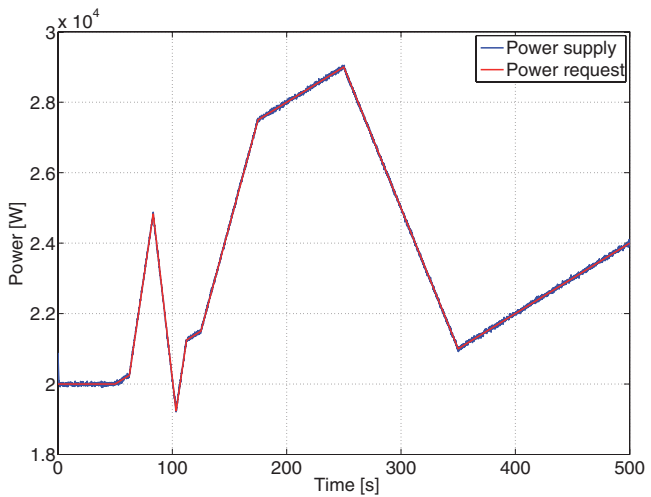

Figure 8

Simulation 2: requested load power.

the minimum values of the solid material distribution along the battery cathode as predicted by the full-order model. The first plot of Figure 11 shows how the controller filters the steps in the power demand, allowing a smoother fuel cell current behavior. An overshoot can be observed at power step demand. This typical second-order system behavior decreases the transient period. The good strategy performance is confirmed by the simulation 2 results, which also highlight the strategy stability for different power request profiles. Furthermore, Figures 12-14 highlight the strategy robustness with respect to the measurement uncertainties. In conclusion, the simulations demonstrate the good behavior of the controlled system. The controller achieves good balancing between the fuel cell and the battery energy supply, aimed at providing the power request and at reaching the nominal operating point.

These simulation results encourage us to continue with testing. In future work we intend to test the control strategy in real time using a dSpace MicroAutoBox Electronic Control Unit (ECU) connected to a dSPACE HIL Simulator 

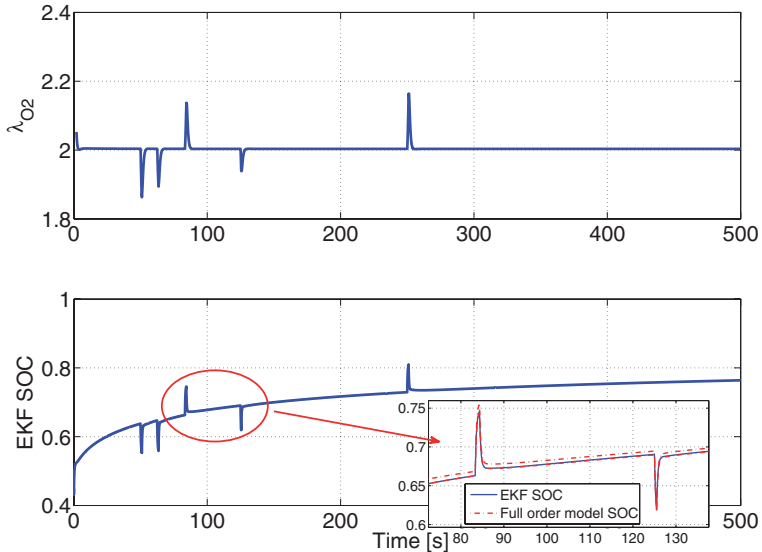

Figure 9

Simulation 1: oxygen ratio and battery State of Charge at cathode. The SOC estimation is compared with the full-order model prediction.
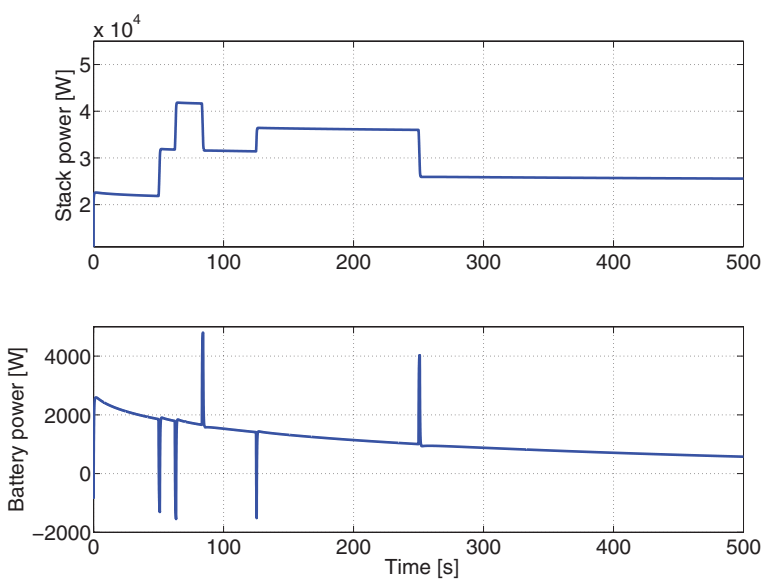

Figure 10

Simulation 1: fuel cell and battery power flow.
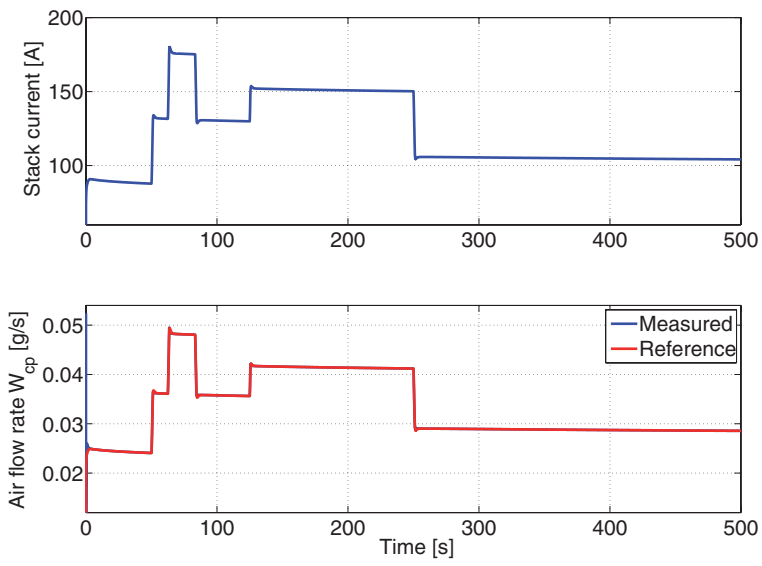

Figure 11

Simulation 1: stack current and compressor air flow rate.
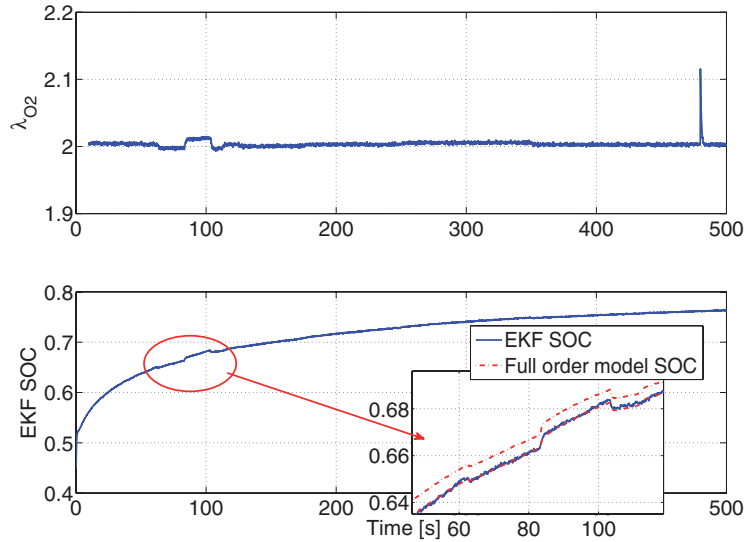

Figure 12

Simulation 2: oxygen ratio and battery State of Charge at cathode. The SOC estimation is compared with the full-order model prediction.
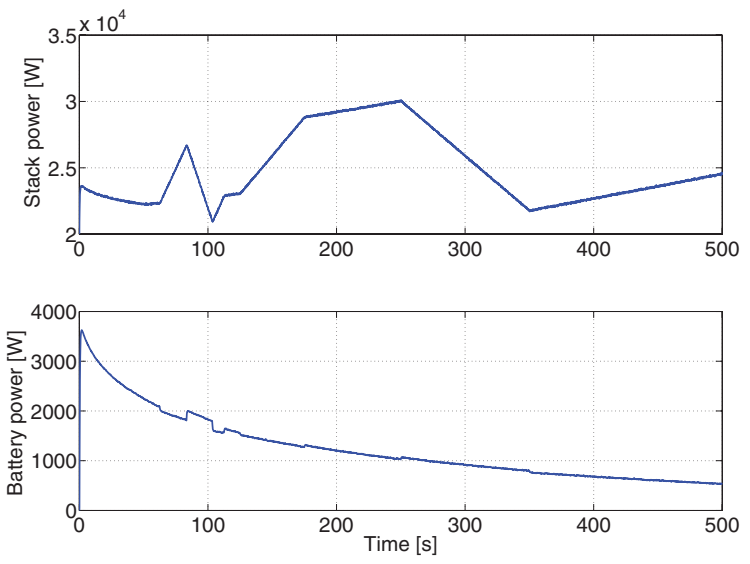

Figure 13

Simulation 2: fuel cell and battery power flow.
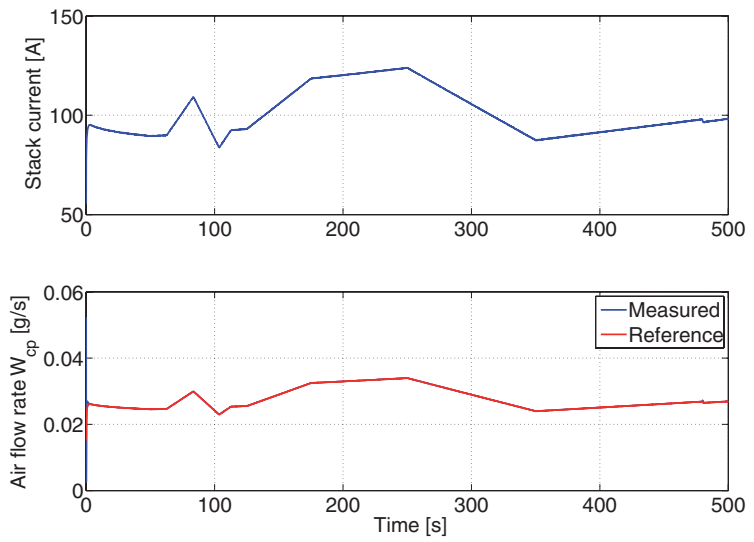

Figure 14

Simulation 2: stack current and compressor air flow rate. 
Mid-Size through a DS2202 I/O board. The dSPACE Simulator will reproduce the FCHPS model while the control strategy will be downloaded in the ECU. This real-time Hardware-in-the-Loop simulation will allow one to test the controller under the ECU prototype feasibility and computational constraints. In order to reduce the computational effort and make the real-time test feasible, we plan to compute off-line a gain scheduling as a function of the power request and stack voltage and to solve the Riccati equation solution $P$ for each point.

\section{CONCLUSION}

The fuel cell performance can be considerably improved with a hybrid configuration which combines a battery through a DC/DC converter. This paper shows a simple decoupled control strategy which allows a good compromise between high performance and safe use of the stack. Here, the fuel cell stack is controlled via a feedforward action and a PI regulator, whereas the DC/DC converter optimizes the current split via a linear quadratic controller.

The strategy was tested in simulation using a 310th-order power system model. Good closed-loop performance was shown.

\section{ACKNOWLEDGEMENTS}

The authors gratefully acknowledge Francesco Vasca for helpful discussions and Anna Stefanopoulou for her invaluable suggestions.

\section{REFERENCES}

1 Anderson B.D.O., Moore J.B. (1990) Optimal Control: Linear Quadratic Methods, Prentice Hall.

2 Barbarisi O., Glielmo L., Vasca F. (2006) State of charge kalman filter estimator for automotive batteries, Control Eng. Pract. 14, 267-275.

3 Domenico D. Di, Fiengo G., Stefanopoulou A. (2008) Lithiumion battery state of charge estimation with a kalman filter based on a electrochemical model, Proceedings of the 2008 IEEE Multi-conference on Systems and Control.

4 Domenico D. Di, Stefanopoulou A., Fiengo G. (2008) Psm: Reduced order lithium-ion battery electrochemical model and extended kalman filter state of charge estimation, submitted to ASME J. Dyn. Syst., Meas. Control - Special Issue on Physical System Modeling.

5 Gao W. (2005) Performance comparison of a fuel cell-battery hybrid powertrain and a fuel cell-ultracapacitor hybrid powertrain, IEEE T. Veh. Technol. 54, 846-855.
6 Guezennec Y., Choi T.-Y., Paganelli G., Rizzoni G. (2003) Supervisory control of fuel cell vehicles and its link to overall system efficiency and low-level control requirements, $A m$. Control Conf. 3, 2055-2061.

7 Soo-Bin Han, Suk-In Park, Hak-Geun Jeoung, Bong-Man Jeoung, Se-Wan Choi (2003) Fuel cell-battery system modeling and system interface construction, The 29th Annual Conference of the IEEE Industrial Electronics Society, Vol. 3, pp. 2623-2627.

8 Jiang Z., Gao L., Dougal R.A. (2005) Flexible multiobjective control of power converter in active hybrid fuel cell/battery power sources, IEEE T. Power Electr. 20, 244-253.

9 Mathworks (2005) Genetic Algorithm and Direct Search Toolbox 2 - User Guide, Mathworks.

10 Nasiri A., Rimmalapudi V.S., Emadi A., Chmielewski D.J., Al-Hallaj S. (2004) Active control of a hybrid fuel cell-battery system, The 4th International Power Electronics and Motion Control Conference, Vol. 2, pp. 491-496.

11 Kim M.-J., Peng H. (2006) Combined control/plant optimization of fuel cell hybrid vehicles, Proceedings of the 2006 American Control Conference, Minneapolis, Minnesota, USA, Vol. 24, pp. 30-46, June 14-16.

12 Pukrushpan J.T., Peng H., Stefanopuolou A.G. (2004) Controloriented modeling and analysis for automotive fuel cell system, J. Dyn. Syst. - T. ASME 126, 14-25.

13 Pukrushpan J.T., Stefanopoulou A.G., Peng H. (2005) Control of Fuel Cell Power Systems: Principles; Modeling; Analysis and Feedback Design, Springer.

14 Rajashekara K., Hybrid fuel-cell strategies for clean power generation, IEEE T. Ind. Appl. 41, 682-689.

15 Smith K., Wang C.Y. (2006) Solid-state diffusion limitations on pulse operation of a lithium-ion cell for hybrid electric vehicles, J. Power Sources 161, 628-639.

16 Stefanopoulou A.G. (2004) Mechatronics in fuel cell systems, Symposium in Mechatronics.

17 Suh K.-W., Stefanopoulou A.G. (2005) Control and coordination of air compressor and voltage converter in load-following fuel cell, Int. J. Energ. Res. 29, 1167-1189.

18 Sun J., Kolmanovsky I.V. (2005) Load governor for fuel cell oxigen starvation protection: A robust nonlinear reference governor approach, IEEE T. Contr. Syst. T. 13, 911-920.

19 Vahidi A., Stefanopoulou A.G., Peng H. (2004) Current management in a hybrid fuel cell power system: A model predictive control approach, IEEE T. Contr. Syst. T. 14, 1047-1057.

20 Wang C.Y., Gu W.B., Liaw B.Y. (1998) Micro-macroscopic coupled modeling of batteries and fuel cells. part i: Model development, J. Electrochem. Soc. 145, 10, 3407-3417.

Final manuscript received in April 2009 Published online in November 2009 or distributed for profit or commercial advantage and that copies bear this notice and the full citation on the first page. Copyrights for components of this work owned by others than IFP must be honored. Abstracting with credit is permitted. To copy otherwise, to republish, to post on servers, or to redistribute to lists, requires prior specific permission and/or a fee: Request permission from Documentation, Institut français du pétrole, fax. +33147527078 , or revueogst@ifp.fr. 